\title{
Patrón espacio-temporal de larvas y huevecillos de nemátodos gastrointestinales en pastizales ganaderos de Veracruz, México
}

\author{
Carolina Flota-Bañuelos ${ }^{1 *}$, Imelda Martínez M. ${ }^{2}$, José López-Collado ${ }^{3}$, \\ Mónica Vargas Mendoza ${ }^{3}$; Héctor González Hernández ${ }^{4} \&$ Pernilla Fajersson ${ }^{3}$ \\ 1. Instituto Tecnológico de Tizimín. Final Aeropuerto Cupul S/N, C.P. 97700, Tizimín, Yucatán; cflota@colpos.mx* \\ 2. Instituto de Ecología, A.C. Red de Ecoetología. Carretera a Coatepec 351, El Haya, Xalapa, C.P. 91070, Veracruz, \\ México; imelda.martinez@inecol.edu.mx \\ 3. Colegio de Postgraduados, Campus Veracruz. Carretera Federal Veracruz-Xalapa Km 88.5 predio Tepetates. C.P. \\ 91690, Veracruz, México; jlopez@colpos.mx, mvargas@colpos.mx, pernillafajersson@hotmail.com \\ 4. Colegio de Postgraduados. Instituto de Fitosanidad. km 35.5 Carr. México-Texcoco, Montecillo, Edo de México. C.P. \\ 56230; hgzzhdz@colpos.mx
}

\author{
Recibido 24-I-2013. Corregido 20-VI-2013. Aceptado 22-VII-2013.
}

\begin{abstract}
Spatio-temporal pattern of larvae and eggs of gastrointestinal nematodes in cattle pastures in Veracruz, Mexico. The spatial and temporal distribution of gastrointestinal nematodes of cattle has been little studied in Mexico. Previous studies have described periods of higher larval presence, vertical and horizontal migration in grasslands, and the frequency of adult nematodes; as well as the effect of pasture trichomes on the migration and survival of Haemonchus larvae. The aim of this study was to determine the time-space layout and spread of gastrointestinal nematode larvae on pasture, and to estimate the effect of ivermectin applied to cattle on the time-dependent abundance of their eggs in a ranch in Veracruz. To determine the spatio-temporal arrangement, monthly morning grass samples were obtained from 30 sampling points from July 2008 to June 2009. Third stage larvae $\left(\mathrm{L}_{3}\right)$ from each point were counted, and aggregation patterns were estimated through variance/mean and negative binomial $\mathrm{K}$ indices. Additionally, the number of eggs per gram in cattle feces was determined, from samples with (CI) and without ivermectin (SI), using standard techniques. A total of $20276 \mathrm{~L}_{3}$ larvae were recovered in the pasture, of which an $80 \%$ corresponded to Haemonchus contortus. The highest nematode density with more than $5000 \mathrm{~L}_{3} / \mathrm{kgDM}$ was detected in October 2008, and the lowest in February and March 2009. The $\mathrm{L}_{3}$ showed an aggregated spatial pattern of varying intensity throughout the year. The number of eggs in the stool was not reduced with the ivermectin application to cattle, which suggested a failure of control. However, the highest parasite loads were observed from July to November 2008. We concluded that the application of ivermectin was not effective to control nematodes eggs, and that $\mathrm{L}_{3}$ populations fluctuated on pasture for ten months, providing an infection source to grazing animals afterwards. Rev. Biol. Trop. 61 (4): 1747-1758. Epub 2013 December 01.
\end{abstract}

Key words: spatial disposition, temporal disposition, Haemonchus contortus, ivermectina, cattle systems.

Los parásitos gastrointestinales que afectan a los bovinos han sido estudiados desde hace varios años, dado que afectan la rentabilidad de los sistemas ganaderos (Escurra, 2001). En la actualidad los antihelmínticos sintéticos son su principal método de control (Waller, 2003), a pesar de que es perjudicial para otras especies de fauna benéfica como los escarabajos estercoleros (Flota-Bañuelos et al., 2012; Cruz et al., 2012). La aplicación de desparasitantes como la ivermectina reduce las poblaciones de nematodos endógenos y esto se refleja en menores cantidades de huevecillos en las heces (Uhlinger et al., 1988). Por lo tanto, la cuantificación de los huevecillos puede proveer una estimación de la efectividad de los desparasitantes, lo cual es deseable, si se pretende mejorar la situación sanitaria del pastizal, por 
tanto una disminución en la cantidad de huevecillos depositados se reflejará en la reducción de larvas infectivas en el pastizal.

Las principales especies de nematodos gastrointestinales (NGI) que atacan a los bovinos en los climas tropicales son: Haemonchus spp., Trichostrongylus spp., y otras de los géneros Cooperia spp. y Oesophagostomum spp. (Olivares et al., 2006). Estas especies de NGI presentan un ciclo de vida de 28 a 35 días, comprendiendo una fase exógena, que inicia con la eliminación de los huevecillos en las heces y termina con la formación de la larva de tercer estadío $\left(\mathrm{L}_{3}\right)$ en el pastizal. La fase endógena (dentro del tracto digestivo del bovino), inicia con la ingestión de las $\mathrm{L}_{3}$ que se encuentran en los pastos por parte de los bovinos (Rege et al., 2002), hasta el desarrollo de los parásitos adultos, la cópula y la producción de huevecillos (Soulsby, 1987). El 5\% del ciclo biológico de los NGI, corresponde a la fase endógena, y el $95 \%$ a la fase exógena (Conder \& Campbell, 1995).

Por lo anterior, la adecuada estimación de poblaciones de $\mathrm{L}_{3}$ en los pastizales, previo al pastoreo de los animales, es esencial para conocer las especies de parásitos y desarrollar estrategias de manejo más apropiadas (Fine et al., 1993, Krecek \& Maingi, 2004), como es el uso de compuestos secundarios de plantas (Vázquez-Luna et al., 2007), la suplementación alimenticia (Coop \& Sykes, 2002) o rotación de potreros (Sani et al., 1995).

La disposición espacial de los organismos es un atributo poblacional que sirve para proponer hipótesis sobre interacciones entre individuos o su respuesta a condiciones ambientales (Vera et al., 2002). Una aplicación potencial del conocimiento de la disposición espacial y de los gradientes poblacionales es orientar los métodos de control hacia los sitios de mayor abundancia, permitiendo ahorrar recursos económicos y eficientizar los agroquímicos $\mathrm{o}$, de otras estrategias de manejo, siendo esto uno de los principales principios de la agricultura de precisión (Blackmore, 2007); también sirve para desarrollar planes de muestreo y así estimar las densidades poblacionales, para la toma de decisiones en la aplicación de control químico o en estudios básicos de biología y ecología de los organismos (Andrews \& Quezada, 1989).

En el caso de la disposición espacial de nematodos, los fitopatógenos han recibido mayor atención, principalmente desde un enfoque epidemiológico. Sobre los NGI del ganado hay menos trabajos reportados, en algunos de ellos se ha determinado la distribución de las larvas de Trichostrongylus en pastizales de bovinos en pastoreo (Gruner \& Sauve 1982), la disposición espacial de Ostertagia en el abomaso de bovinos (Morales et al., 1991), la distribución y migración de las larvas de Trichostrongylus tenius (Saunders et al., 2000). En México solo se conocen los horarios de mayor presencia larvaria, las migraciones verticales y horizontales en los pastizales (Sandoval et al., 2002) y la frecuencia de nematodos adultos (Vázquez et al., 2004). En climas tropicales similares a Veracruz, se ha estudiado el efecto del tricoma de los pastos sobre la migración y supervivencia de larvas de Haemonchus (Oliveira et al., 2009).

El objetivo de este trabajo fue conocer la disposición espacio-temporal y diseminación de las larvas de nematodos gastrointestinales en los pastizales, y estimar el efecto de la ivermectina aplicada a bovinos sobre la abundancia de sus huevecillos.

\section{MATERIALES Y MÉTODOS}

Ubicación: Los estudios se realizaron simultáneamente en el Rancho San Ramón, ubicado en Medellín de Bravo, Veracruz, México, a $18^{\circ} 58^{\prime} 19^{\prime \prime} \mathrm{N}-96^{\circ} 04^{\prime} 51^{\prime \prime} \mathrm{W}$ y una altitud de $52 \mathrm{msnm}$. El clima de la zona es cálido húmedo, con una temperatura promedio anual de $25.3^{\circ} \mathrm{C}$. La precipitación pluvial media anual es de $1417.8 \mathrm{~mm}$. El suelo es de tipo arenoso y se caracteriza por tener una capa superficial rica en materia orgánica (García, 1987).

Se seleccionó un pastizal relativamente plano de aproximadamente $1.5 \mathrm{ha}$, delimitado por cercas vivas de Gliricidia sepium, Guazuma ulmifolia, Cordia dodecandra y 
Byrsonima crassifolia, con pasto estrella de África (Cynodon nlemfuensis) y pasto Guinea (Panicum maximun). El potrero fue utilizado de manera rotacional con cinco días de ocupación por los 26 bovinos seleccionados, y 30 días de descanso, para la recuperación del pasto y para que estén presentes las $\mathrm{L}_{3}$, presentando una carga animal de 2.8 unidades animal por hectárea (UA/ha).

Disposición espacial de larvas de nematodos en el pastizal: Los muestreos de pasto se realizaron mensualmente de julio 2008 a junio 2009, utilizando la técnica de Taylor (1939) con modificaciones. Se seleccionaron sistemáticamente 30 puntos fijos para el muestreo, que fueron numerados y marcados con varillas de madera. Los puntos de muestreo tenían $40 \mathrm{~m}$ de separación entre ellos, para poder abarcar la mayor cantidad del área del pastizal. Los muestreos de larvas se realizaron en horario de 5:007:00h, siguiendo la técnica de Bryan \& Kerr (1988) y 15 días después del pastoreo de los bovinos, dando tiempo a que los huevecillos de los nematodos eclosionaran y maduraran en $\mathrm{L}_{3}$.

En cada punto marcado se colocó un cuadrante de plástico de $15 \times 15 \mathrm{~cm}$ sobre el pasto. Se cortó todo el pasto dentro del cuadrante a $5 \mathrm{~cm}$ del suelo y fue colocado en bolsas de plástico previamente rotuladas con el número, hora y punto de muestreo. Posteriormente, el pasto se depositó en una charola de plástico con $10 \mathrm{~L}$ de agua potable y se dejó reposar por $3 \mathrm{~h}$. Después se agitó el pasto, y el agua resultante se filtró por tres tamices con mallas de 50, 100 y $400 \mu$ de abertura. Las larvas de tercer estadio obtenidas en la malla de $400 \mu$, se contaron (Niec, 1972) e identificaron, empleando la clave taxonómica descrita por Niec (1968). El pasto se pesó y se secó en una estufa a una temperatura de $60^{\circ} \mathrm{C}$ por $48 \mathrm{~h}$, para determinar el porcentaje de materia seca. Para el cálculo del índice de infestación del pasto se empleó la fórmula: $L_{3} / K g M S=\left(N L_{3} / K g M S\right) \times 1000$, donde: $\mathrm{L}_{3} / \mathrm{KgMS}$ : Número de larvas infectantes por kilogramo de materia seca, $\mathrm{N} \mathrm{L}_{3}$ : Número de larvas contadas y MS: Materia seca del pasto en kilogramos.
Conteo de huevecillos de nematodos gastrointestinales en las heces bovinas: Del total de 50 bovinos, se seleccionaron 26 bovinos F1 (cruzas suizo x cebú), los cuales se dividieron en dos grupos de 13 animales de acuerdo a su carga parasitaria. En el grupo CI, los 13 bovinos fueron desparasitados con la dosis recomendada por el fabricante, y que fue aplicada en junio y diciembre 2008, y junio 2009. Cada bovino recibió $1 \mathrm{~cm}^{3}$ de Iverject F. Bovinos ${ }^{\circledR}$ vía intramuscular por cada $100 \mathrm{~kg}$ de peso vivo. En el grupo SI, los 13 bovinos no se desparasitaron durante el período de estudio.

La recolección de heces se realizó mensualmente de julio 2008 a junio 2009, se obtenieron $100 \mathrm{~g}$ de estiércol fresco directamente del recto de cada animal. Cada muestra se colocó en bolsas de polietileno previamente marcadas con el número de cada animal. El estiércol de cada animal se homogeneizó y posteriormente fue procesado individualmente empleando la técnica de McMaster (Rodríguez et al., 1994), usando una solución glucosada con 1.270 de densidad y una cámara de McMaster para cuantificar los huevecillos (Thienpond et al., 1986).

Los datos meteorológicos (medias de precipitación y temperatura), fueron proporcionados por la estación meteorológica del campo experimental Cotaxtla, Veracruz, perteneciente al Instituto Nacional de Investigaciones Forestales, Agrícolas y Pecuarias (INIFAP).

Para conocer la disposición espacial de las larvas en el pastizal, se utilizaron los datos obtenidos de las distribuciones de los géneros de larvas en cada sitio y fecha de muestreo. Se calculó el patrón de disposición espacial mediante los índices de dispersión: varianza/ media $V M=S^{2} / \bar{Y}$, que toma valores de 1 para una disposición aleatoria, valores $<1$ para un arreglo regular $\mathrm{y}>1$ para poblaciones agregadas (Taylor, 1961) y K de la distribución binomial negativa, $K=Y^{2} /\left(S^{2}-\bar{Y}\right)$, con valores $>8$ para poblaciones con disposición aleatoria, y entre 0 y 8 para poblaciones con disposición agregada (Rojas, 1964). Finalmente, se generaron mapas de iso-densidades a partir de un conjunto de observaciones. Los mapas se obtuvieron, dividiendo el pastizal en celdas y a 
cada celda se le asignó un valor estimado con los puntos de muestreo. El valor numérico de cada celda se convirtió en un color mediante una escala de colores, donde cada color representó un intervalo en el número de larvas. La estimación de índices se hizo acorde con Pielou (1960) para la relación varianza-media y se probó la hipótesis nula $H o: S^{2}=\bar{Y}$ ) con una prueba de chi-cuadrada (Steel et al., 1997), $\mathrm{K}$ se estimó por momentos (Bliss \& Fisher, 1953); las isodensidades se calcularon mediante interpolación lineal inversa. Los cálculos de índices y mapas se hicieron con el programa SUPRA (López-Collado, 2004).

Para evaluar el efecto de la ivermectina sobre la abundancia de huevecillos en las heces, se utilizó un modelo lineal mixto para mediciones repetidas en el tiempo; el modelo incluyó el efecto de los tratamientos, el mes de muestreo y su interacción. Se usó el procedimiento MIXED y una estructura de covarianzas AR(1), seleccionada iterativamente de acuerdo con los criterios de información de Akaike y por el criterio de información bayesiano (Litell et al., 1998). Los análisis se hicieron con SAS v9.1 (SAS Institute, 2004). Con este modelo se comparó el efecto de los tratamientos en el tiempo. Este mismo procedimiento se aplicó para evaluar las poblaciones de larvas infectantes en el pastizal, pero en este caso el modelo incluyó solamente el tiempo como factor de estudio y se determinó si habían o no diferencias en las fechas de muestreo.

\section{RESULTADOS}

Se obtuvieron en total 20276 larvas infectantes durante los doce meses de muestreo en el pastizal. Las larvas encontradas pertenecen a cuatro especies: Haemonchus contortus, Cooperia sp., Trichostrongylus axei y Ostertagia sp. del orden Strongylida y la superfamilia Trichostrongyloidea. La mayor abundancia fue de $H$. contortus con más del $80 \%$ durante el período de muestreo. Mientras que las tres especies Cooperia sp., Trichostrongylus axei y Ostertagia sp., juntas representaron sólo el 20\% (Fig. 1).
La única especie que se observó durante todo el período de estudio fue $H$. contortus, las demás especies únicamente se presentaron de cuatro a seis meses (Fig. 1).

En la densidad total de larvas por mes de las cuatro especies recuperadas en el pastizal, se encontraron diferencias significativas $\left(\mathrm{F}_{9,290}=22.17, \mathrm{p}<0.001\right)$. En octubre 2008 se recolectaron más de $5000 \mathrm{~L}_{3} / \mathrm{kg} \mathrm{MS}$, mientras que de enero a marzo 2009, se encontraron las menores cantidades de larvas infectivas, menos de $2000 \mathrm{~L}_{3} / \mathrm{kg}$ MS (Fig. 2A). En abril y mayo 2009, que son los meses más secos del año en el área de estudio, los bovinos consumieron el escaso forraje que se encontraba en el pastizal, y por tanto no hubo material vegetal para el muestreo (Fig. 2B). Por otra parte, la abundancia total de las $\mathrm{L}_{3}$ de las cuatro especies de NGI, se correlacionó positivamente con la precipitación $\left(\mathrm{r}_{\mathrm{s}}=0.61, \mathrm{p}=0.035\right)$, se encontraron más larvas infectantes cuando se registró mayor precipitación y la menor abundancia de nematodos, cuando disminuyó la precipitación (Fig. 2B).

En cuanto al análisis espacial de las larvas infestantes, presentaron un patrón agregado, señalado por los índices de Varianza media. Sin embargo, la K-binomial negativa muestra agregación solamente en enero y marzo 2009 (Cuadro 1). El mes con mayor agregación indicado por la relación varianza/media fue marzo 2009, y de julio a septiembre 2008 y febrero 2009, tuvieron menor agregación (Cuadro 1).

Los mapas espacio-temporales, muestran el arreglo in situ de las larvas infectivas, señalando su disposición durante 12 meses (Fig. 3). La mayor densidad se presentó en octubre 2008 , en contraste con enero, febrero y marzo 2009 , donde se observaron las menores densidades poblacionales de larvas de NGI (Fig. 3).

De igual manera, se observa que las larvas de NGI se encontraron en todo el potrero durante todos los meses (Fig. 3). Sin embargo, en febrero 2009, se observó una población menor a $90 \mathrm{~L}_{3}$ por punto de muestreo, que está relacionado con el mes de menor precipitación (Fig. 2B).

En cuanto al efecto de la ivermectina sobre la abundancia de huevecillos de NGI, no se encontró interacción tratamiento*tiempo 


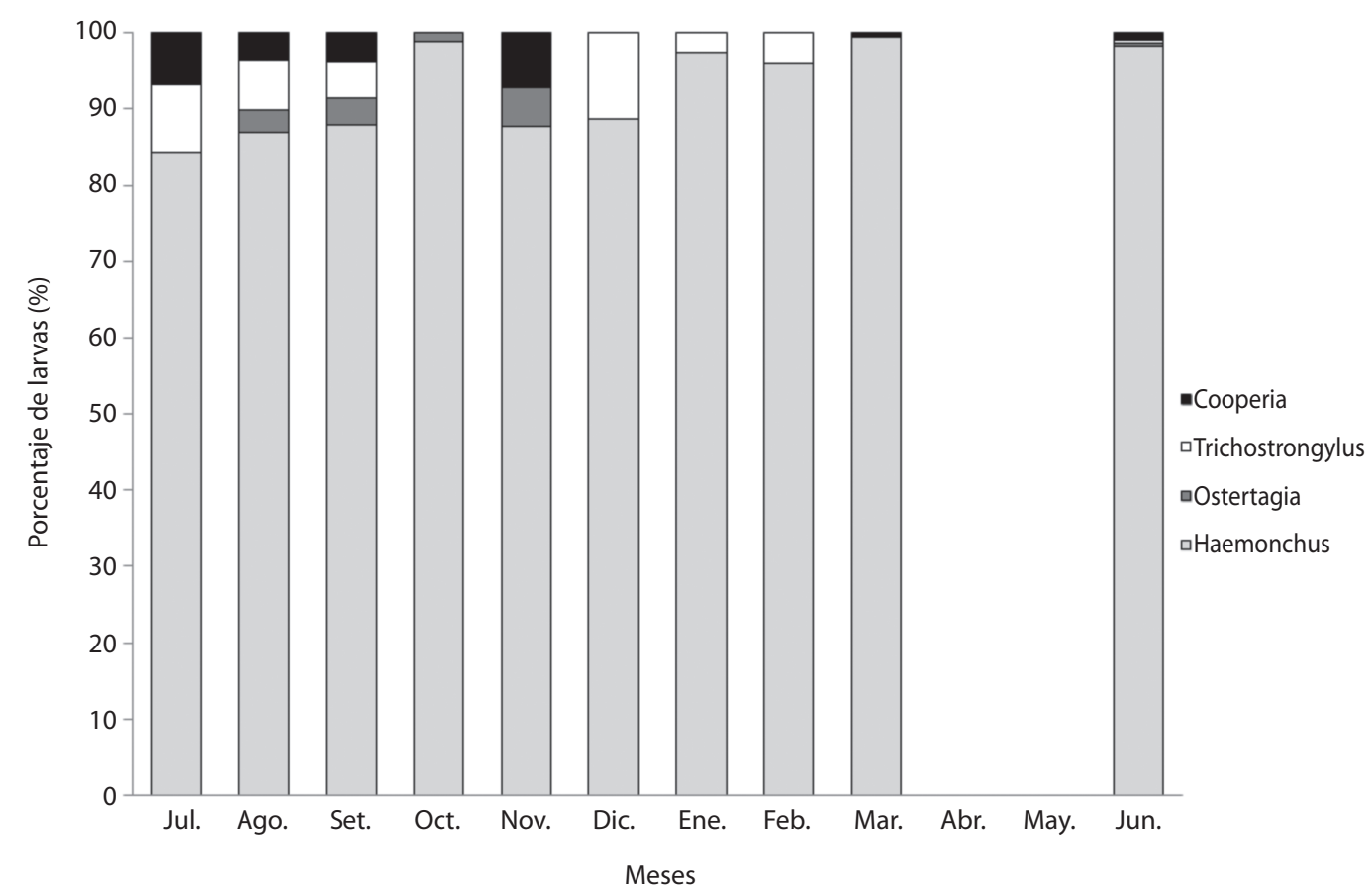

Fig. 1. Prevalencia de larvas de tercer estadio (Haemonchus contortus, Ostertagia sp., Trichostringylus axei y Cooperia sp.) presentes en un pastizal de Medellín, Veracruz.

Fig. 1. Prevalence of third instar larvae (Haemonchus contortus, Ostertagia sp., Trichostringylus axei y Cooperia sp.) present in a pasture of Medellin, Veracruz.

CUADRO 1

Índices de disposición espacial de Haemonchus contortus en un pastizal de Veracruz, México

TABLE 1

Indices of spatial disposition of Haemonchus contortus in a pasture of Veracruz, Mexico

\begin{tabular}{lcc}
\multicolumn{1}{c}{ Meses } & \multicolumn{2}{c}{ Índices } \\
& Varianza-media & K binomial negativa \\
Julio & $4.1^{*}$ & 24.9 \\
Agosto & $2.2^{*}$ & 66.7 \\
Septiembre & $1.4^{*}$ & 167.2 \\
Octubre & $7.3^{*}$ & 17.7 \\
Noviembre & $7.8^{*}$ & 9.6 \\
Diciembre & $7.1^{*}$ & 11.2 \\
Enero & $13.6^{*}$ & $3.3^{*}$ \\
Febrero & $2.3^{*}$ & 25.1 \\
Marzo & $18.2^{*}$ & $1.8^{*}$ \\
Abril & -- & -- \\
Mayo & -- & -- \\
Junio & $8.4^{*}$ & 8.2 \\
\hline
\end{tabular}

*Significativamente agregada (prueba de $\chi^{2}, \mathrm{p}<0.05$ ), no hubo datos.
$\left(\mathrm{F}_{11,251}=0.77, \mathrm{p}=0.6691\right)$, ni efecto del tratamiento durante todo el tiempo de muestreo $\left(\mathrm{F}_{1,251}=0.92, \mathrm{p}=0.3376\right)$. Sin embargo, se observaron diferencias significativas entre las cantidades de huevecillos encontrados en los diferentes meses $\left(\mathrm{F}_{11,251}=8.97, \mathrm{p}<0.0001\right)$. Julio $\left(\mathrm{F}_{1,251}=8.99, \mathrm{p}<0.0001\right)$, octubre $\left(\mathrm{F}_{1,251}=6.39\right.$, $\mathrm{p}<0.0001)$ y noviembre $2008 \quad\left(\mathrm{~F}_{1,251}=6.18\right.$, $\mathrm{p}<0.001$ ), fueron los que presentaron mayor número de huevecillos por gramo de heces (Fig. 4). También se observó que durante los doce meses de estudio, la carga parasitaria de todos los bovinos se mantuvo por debajo de 180hpg.

\section{DISCUSIÓN}

La mayor cantidad de $\mathrm{L}_{3}$ de todas las especies de NGI en el pastizal se obtuvo en octubre, lo que posiblemente se debió a las 

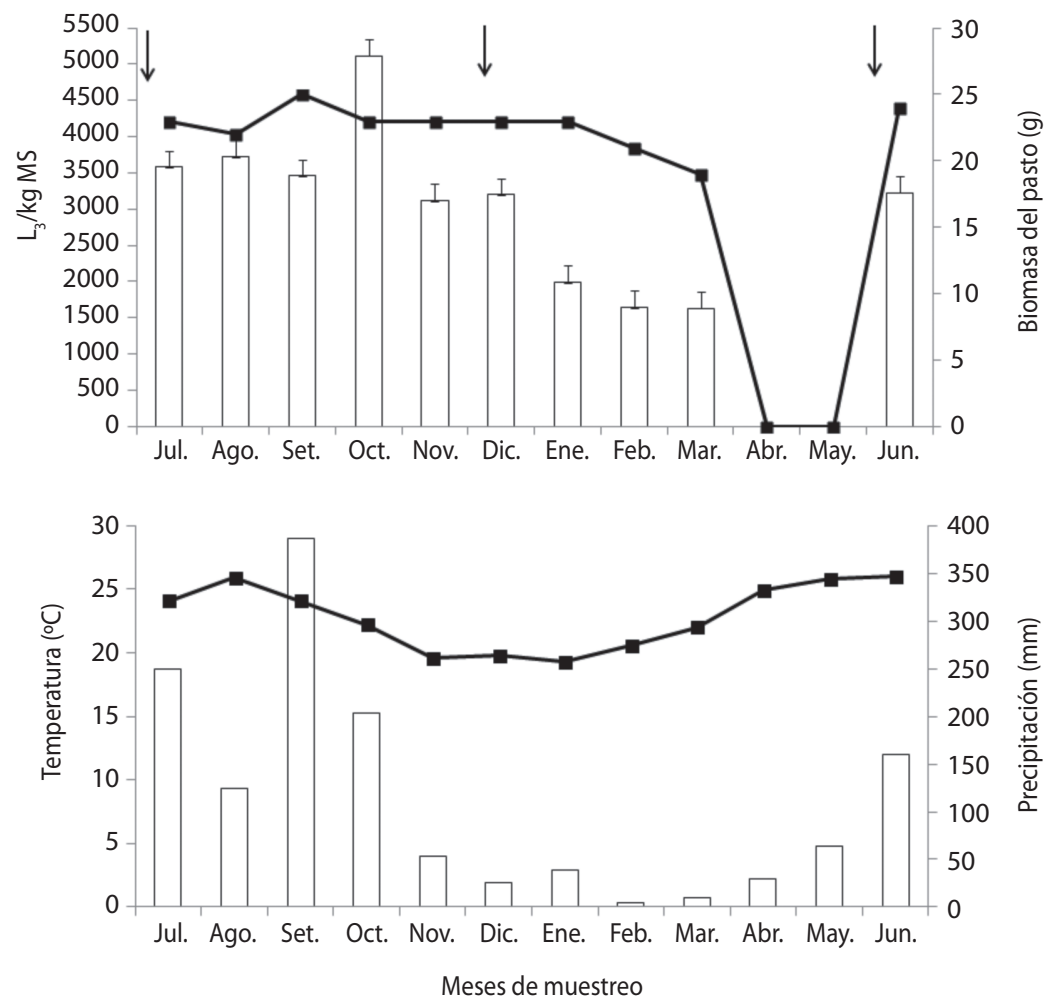

Fig. 2. (A) Abundancia de larvas infectantes de nematodos gastrointestinales ( $\square$ ) por kilogramos de biomasa seca de pasto $\left(\mathrm{L}_{3} \mathrm{kgMS}\right)(-\mathbf{-})$. (B) Media de temperatura $(-\mathbf{-})$ y precipitación $(\square)$ de julio 2008 a junio 2009 (Fuente: Estación meteorológica del INIFAP Cotaxtla, Veracruz). Las flechas indican aplicación de ivermectina a los bovinos.

Fig. 2. (A) Abundance of gastrointestinal nematode infective larvae ( $\square$ ) per kg of dry biomass of grass $\left(\mathrm{L}_{3} / \mathrm{kgMS}\right)(-\mathbf{-})$. (B) Average temperature (-) and rainfall ( $\square$ ) from July 2008 to June 2009 (Source: Meteorological Station Cotaxtla, Veracruz INIFAP). The arrow indicates application of ivermectin to cattle.

precipitaciones más altas registradas en septiembre 2008 con más de $350 \mathrm{~mm}$ en promedio. En este sentido, Levine (1963) señala que con un mínimo de $50 \mathrm{~mm}$ de precipitación, los huevecillos de $H$. contortus eclosionan y posteriormente las larvas mudan a $\mathrm{L}_{3}$ en aproximadamente tres semanas.

Lo anterior también coincide con lo estudiado por Liebano et al., (1998), quienes reportan más larvas de $H$. contortus en Jiutepec, Morelos, de octubre a diciembre, que correspondió a la época de lluvias, mientras que las menores cantidades de larvas se observaron de enero a marzo, cuando se presentan las precipitaciones más bajas. El mismo comportamiento de distribución estacional fue reportado por
Fernández et al. (1994), quienes no encontraron ninguna larva de $H$. contortus en diciembre 1989, febrero y marzo 1990, que correspondió a los meses más secos en Jiutepec, Morelos.

En el pastizal experimental de estudio, en julio, agosto y septiembre 2008, y junio 2009 no se detectaron la mayores cantidades de larvas de NGI, sin embargo, fueron meses que presentaron más de $100 \mathrm{~mm}$ de precipitación en promedio, los cuales pudieron favorecer las eclosiones de los huevecillos y por consiguiente, aumentar la infestación del pastizal en un lapso no mayor de tres semanas, como lo sugieren Rossanigo \& Gruner (1994). En este sentido, Iqbal et al. (2005) señalan que la mayor contaminación de los pastizales en 

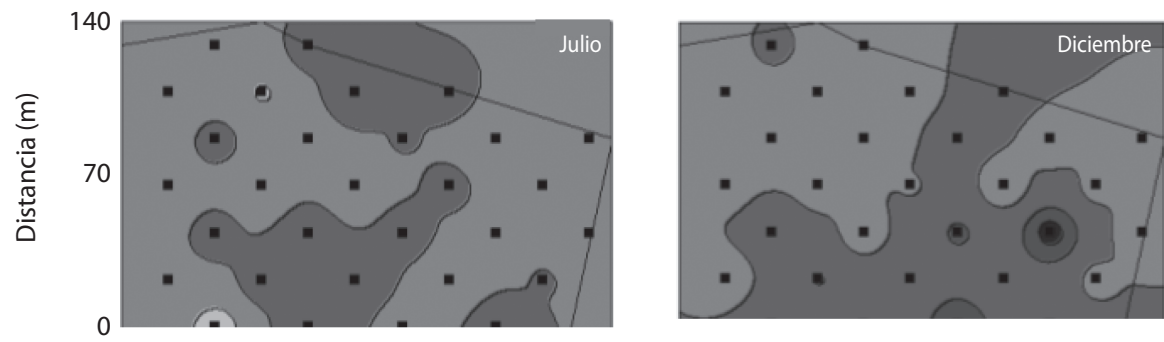

Número de larvas 3
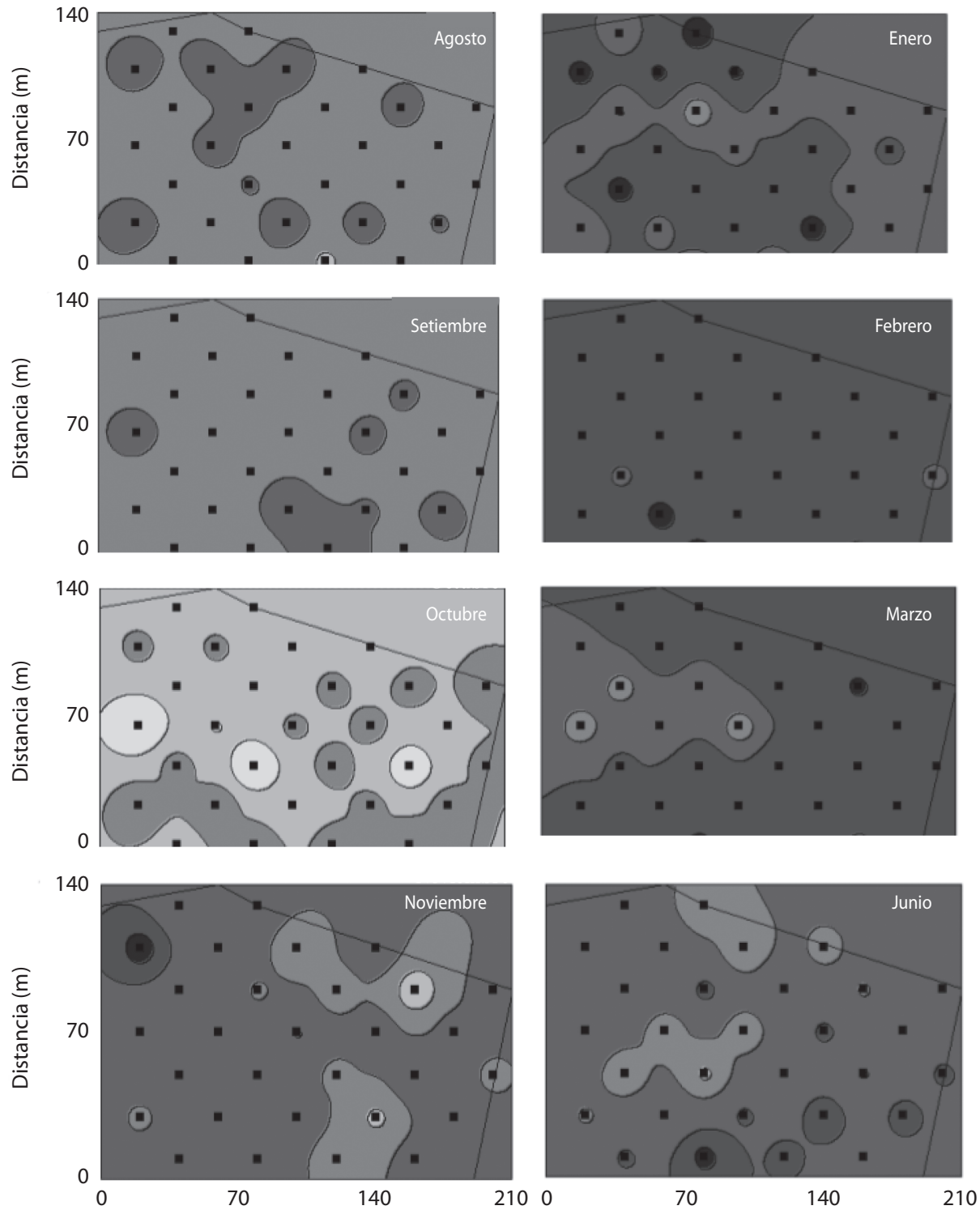

Fig. 3. Isodensidades del número total de $\mathrm{L}_{3}$, recolectadas en un pastizal de Medellín, Veracruz. El símbolo cuadrado indica un punto de muestreo de las larvas.

Fig. 3. Isodensity $\mathrm{L}_{3}$ total number collected in a pasture of Medellin, Veracruz. The square symbol indicates a sampling point of the larvae. 


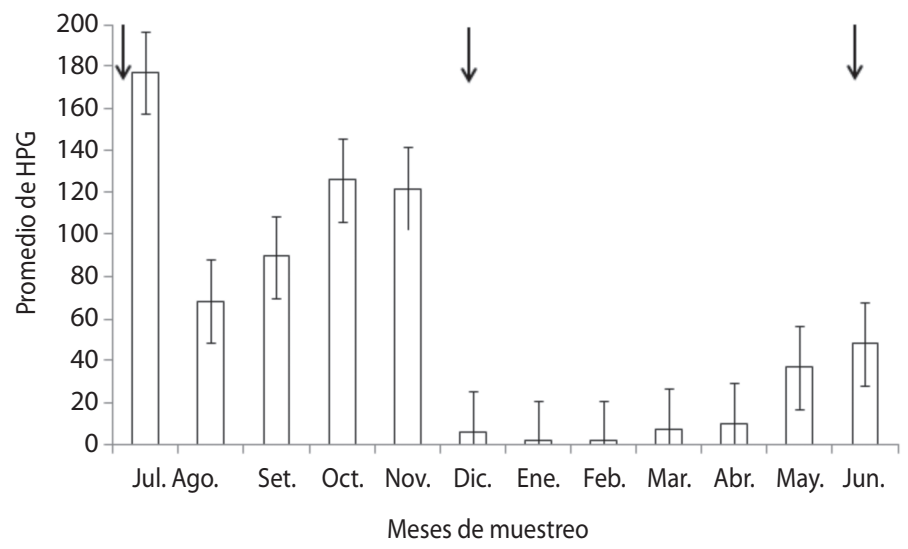

Fig. 4. Media de huevecillos por gramo de heces (hpg) de bovinos en pastoreo. Las flechas indican aplicación de ivermectina a los bovinos.

Fig. 4. Average number of gastrointestinal nematode eggs per gram of feces (hpg) of cattle, obtained from July 2008 to June 2009.

Pakistán es de julio a septiembre, lo que se relaciona con la época de monzones. Por su parte, Encalada et al. (2009) mencionan que los picos de infección de las pasturas en Escárcega, Campeche, se presentan de junio a octubre, que son los meses con mayor precipitación. De igual manera, Silva et al. (2010) indican que la precipitación favorece la migración larvaria, por lo que a mayor precipitación y humedad, mayor cantidad de larvas en los pastizales. En este sentido, Altaif \& Jacobs (1987) y Arece \& Rodríguez (2010) en Iraq y Cuba, respectivamente, atribuyen que la dinámica de las $\mathrm{L}_{3}$ en el pasto se debe a los efectos positivos de las precipitaciones, la temperatura y la radiación solar.

La especie $H$. contortus fue la más abundante, con una presencia de $80 \%$ del total de los meses de muestreo, incluyendo períodos de sequía. Al respecto, Amaradasa et al. (2010) señalan que $H$. contortus, puede sobrevivir relativamente en periodos secos por más de 21 días. Nari et al. (1977) encontraron un pico de parasitismo en borregos de 6 a 12 meses causado por $H$. contortus de junio a septiembre, previo a un estado hipobiótico de las larvas, haciendo que sobrevivan a períodos desfavorables desde el punto de vista climático, y reactivándose posteriormente de forma masiva.
Se determinó que las larvas de $H$. contortus son más abundantes de junio a noviembre, comprendidos en el verano y el otoño, mientras que Cooperia, Trichostrongylus y Ostertagia, fueron abundantes en (julio y noviembre), (julio y diciembre) y (noviembre) respectivamente. Similar a lo estudiado por Krecek et al. (1991) en Sudáfrica. Así mismo, Giudici et al. (1999) mencionan que el bajo porcentaje de Trichostrongylus y la abundancia de H. contortus, coincide con lo indicado para climas cálidohúmedos. Jimenez et al. (2007) en Costa Rica, encontraron que las larvas de Haemonchus spp. y Cooperia spp. son más predominantes de mayo a agosto, mientras que Trichostrongylus spp. y Oesophagostomum spp. fueron menos prevalentes. Las condiciones climatológicas tanto de temperatura como precipitación, adaptación a la sequía, así como la capacidad hipobiótica de $H$. contortus, hacen que esta especies tenga mayor prevalencia durante todo el año, a diferencia de las otras especies que están más adaptadas a temperaturas por debajo de $\operatorname{los} 25^{\circ} \mathrm{C}$ y una precipitación arriba de $100 \mathrm{~mm}$ (Banks et al., 1990). Estos estudios y otros realizados en ovinos, concuerdan con la dominancia y competitividad en los pastizales por parte de las $\mathrm{L}_{3}$ de $H$. contortus comparada con 
T. columbriformis y Oesophagostomum (Bailey et al., 2009, Encalada et al., 2009).

El análisis espacial mostró un patrón agregado de las $\mathrm{L}_{3}$ infectantes en el pastizal durante los diez meses de muestreo. En este sentido Boag et al. (1992) mostraron que H. contortus y otras especies de NGI como Nematodirus battus, Teladostargia circumcincta, Chabertia ovina, Trichuris ovis, Strongyloides papillosus, T. axei, Cooperia curticei y $O$. venulosum, presentan un patrón de agregación en diferentes meses del año.

Lo anterior también coincide con lo reportado por Boag et al. (1989) quienes al estudiar la distribución espacial de las larvas infectantes de NGI (Nematodirus battus, N. filicollis y $T$. vitrinus) en pastizales con ovinos, encontraron un patrón agregado, similar a la especie $T$. tenius evaluada en condiciones de campo por Saunders et al. (2000). Este comportamiento puede deberse a que la oviposición de huevecillos de NGI es en masas con una posterior dispersión de las larvas. Este tipo de procesos son factibles de representarse por conglomerados distribuidos al azar (Neyman, 1939). En este contexto, las larvas infectivas presentaron una disposición agregada, aunque los huevecillos pudieron haber sido depositados aleatoriamente dentro de las heces por el ganado en pastoreo. También pudo deberse a otros factores como lo descritos por Boom \& Sheath (2008), quienes al evaluar la dinámica poblacional de las $\mathrm{L}_{3}$ en el pasto, encontraron que la poda de pasto y la presencia de una lluvia significativa arriba de $100 \mathrm{~mm}$, hacen que las $\mathrm{L}_{3}$ se agreguen hacia el estiércol una vez eclosionados los huevecillos.

Por otra parte, la desparasitación de los bovinos con ivermectina, realizada en julio y diciembre 2008 y en junio 2009, no tuvo efecto en la disminución de los huevecillos por gramo de heces, debido a que se observó el mayor número de éstos durante julio, octubre y noviembre 2008 en los dos grupos, CI y SI. En este sentido, Tyrrell et al. (2002) demostraron que la ivermectina aplicada oralmente a ovinos no disminuye el número de huevecillos de $H$. contortus en el estiércol, por el contrario, aumentaron a partir del décimo día de la aplicación. Sin embargo, existen estudios que demuestran la eficacia de la desparasitación con ivermectina, como el realizado por Francisco et al. (2011) quienes al evaluar dos dosis de ivermectina (T1: $0.0005 \mathrm{~cm}^{3} / \mathrm{kg}$ peso vivo y T2: $0.001 \mathrm{~cm}^{3} / \mathrm{kg}$ peso vivo) en caballos en pastoreo, sobre la reducción de huevecillos, concluyeron que el uso de $0.001 \mathrm{~cm}^{3} / \mathrm{kg}$ peso vivo controla satisfactoriamente la infestación por nematodos gastroentéricos.

Es importante señalar que la cantidad de huevecillos por gramo de heces que presentaron los bovinos durante todo el estudio, estuvo por debajo de los $180 \mathrm{hpg}$, estando los animales, dentro del nivel de infección leve que corresponde al conteo de 50 a 200 hpg (Morales \& Pino, 2009). Sin embargo, son cantidades suficiente de huevecillos que son depositados al pastizal, para que se presenten larvas infectivas durante todo el año.

En el presente estudio posiblemente los nematodos estudiados han desarrollado resistencia a la ivermectina, la cual ha sido aplicada constantemente por más de 15 años en el rancho donde se realizaron los experimentos. La resistencia se expresa cuando después de cada tratamiento sobrevive un pequeño número de individuos resistentes al químico utilizado, éstos podrán reproducirse y contaminar los pastizales con sus huevecillos (Jackson, 1993).

Se puede concluir, que en el presente estudio la aplicación de ivermectina en los bovinos no disminuyó la cantidad de huevecillos de nematodos gastrointestinales. Siendo innecesaria su aplicación. Así mismo, la presencia de $\mathrm{L}_{3}$ de $H$. contortus en el pastizal durante diez meses, provoca una constante contaminación del pastizal, debido a que los animales salen al pastoreo durante las primeras horas del día, cuando las larvas se encuentran realizando migraciones en el pasto; lo cual se reflejó en la disposición espacial de las larvas, que se presentaron en todo el pastizal estudiado.

Se recomienda el desarrollo y uso de modelos que simulen la distribución de las especies de nematodos, con base en la representación de los procesos fisiológicos, ambientales y antropogénicos que la determinan, utilizando 
herramientas de dinámica de sistemas, que ayuden a comprender integralmente al fenómeno y aportar escenarios capaces de validarse en los sistemas ganaderos, para volverse una guía en la toma de decisiones (Candelaria et al. 2011).

\section{AGRADECIMIENTOS}

Al Colegio de Postgraduados por el financiamiento de tesis mediante el Fideicomiso $\mathrm{N}^{\circ}$ 167304 para el Establecimiento y Operación de los Fondos para la Investigación Científica y Desarrollo Tecnológico del Centro Público Colegio de Postgraduados. Al CONACYT con la beca $\mathrm{N}^{\circ} 164003$ otorgada al primer autor. $\mathrm{Al}$ Instituto de Ecología A. C. de Xalapa, Veracruz, México y a la Universidad VeracruzanaFacultad de Medicina Veterinaria y Zootecnia.

\section{RESUMEN}

El conocer la disposición espacio-temporal y diseminación de las larvas de nematodos gastrointestinales en los pastizales, y estimar el efecto de la ivermectina aplicada a bovinos sobre la abundancia de sus huevecillos, permite estimar dónde y cuándo se presentan las poblaciones más altas, que puede servir para establecer planes de muestreo y orientar medidas de control, así como para definir el riesgo de contaminación de los pastizales de manera diferencial. Para la determinación de la disposición espacio-temporal de $\mathrm{L}_{3}$ en el pastizal, se recolectaron, contaron e identificaron mensualmente las larvas en 30 puntos de muestreo, posteriormente se generaron mapas de disposición espacial con los datos obtenidos de los conteos de $\mathrm{L}_{3}$ en cada punto y mes de muestreo, y se calculó el patrón de disposición mediante los índices varianza/media y $\mathrm{K}$ binomial negativa. El número de huevecillos por gramo de heces de los bovinos con (CI) y sin ivermectina (SI), se calculó con la técnica de McMaster. En el pastizal se recuperaron 20 $276 \mathrm{~L}_{3}$, correspondiendo el $80 \%$ a $H$ contortus. En octubre 2008 se detectó la más alta densidad de nematodos con más de $5000 \mathrm{~L}_{3} / \mathrm{kgMS}$. Las $\mathrm{L}_{3}$ presentaron un patrón espacial agregado de intensidad variable durante todo el año. Las mayores densidades poblacionales de nematodos fueron en octubre 2008 y las menores en febrero y marzo 2009. La aplicación de ivermectina a los bovinos no redujo el número de huevecillos presentes en las heces, debido a que los tratamientos fueron estadísticamente iguales. De julio a noviembre 2008, se observaron las mayores cargas parasitarias.

Palabras clave: disposición espacial, disposición temporal, Haemonchus contortus, ivermectina, sistemas ganaderos.

\section{REFERENCIAS}

Altaif, K. L., \& Jacobs, A. Y. (1987). Development and survival of Haemonchus contortus larvae on pasture in Iraq. Tropical Animal Production, 19, 88.

Amaradasa, B. S., Lane, R. A., \& Managent, A. (2010). Vertical migration of Haemonchus contortus infective larvae on Cynodon dactylon and Paspalum notatum pastures in response to climatic conditions. Veterinary Parasitology, 170, 78-87.

Andrews, L. K., \& Quezada, J. R. (1989). Manejo integrado de plagas insectiles en la agricultura: Estado actual y futuro. Escuela agrícola Panamericana. El Zamorano, Honduras, Centroamérica.

Arece, J., \& Rodríguez, J. G. (2010). Dinámica de las larvas infestantes de estrongílidos gastrointestinales en ovinos en pastoreo. Pastos y Forrajes, 33(1), 1-17.

Bailey, J. N., Kahn, L. P., \& Walkden-Brown, S. W. (2009). Availability of gastrointestinal nematode larvae to sheep following winter contamination of pasture with six nematode species on the Northern tablelands of New South Wales. Veterinary Parasitology, 160, 89-99.

Banks, D. J. D., Singh, R., Barger, I. A., Pratap, B., \& LeJambre, L. F. (1990). Development and survival of infective larvae of Haemonchus contortus and Trichostrongylus columbriformis on pasture in a tropical environment. International Journal for Parasitology, 20, 155-160.

Blackmore, S. (2007). Agricultura de Precisión (AP). Revista Nacional de Agricultura, 949, 20-28.

Bliss, C. I., \& Fisher, R. A. (1953). Fitting the negative binomial distribution to biological data and note the efficient fitting of the negative binomial. Biometrics, 9, 176-200.

Boag, B., Topham, P. B., \& Webster, R. (1989). Spatial distribution on pasture of infective larvae of the gastrointestinal nematode parasites of sheep. International Journal of Parasitology, 19, 681-685.

Boag, B., Hackett, C. T., \& Topham, P. B. (1992). The use of Taylor's power law to describe the aggregated of gastrointestinal nematodes of sheep. International Journal of Parasitology, 22, 267-270.

Boom, C. J., \& Sheath, G. W. (2008). Migration of gastrointestinal nematode larvae from cattle faecal pats onto grazable herbage. Veterinary Parasitology, 157, 260-266.

Bryan, R. P., \& Kerr, J. D. (1988). The grazing behavior of cattle in relation to the sampling of infective nematode larvae on pasture. Veterinary Parasitology, 30, 73-82.

Candelaria, M. B., Ruiz, R. O., Gallardo, L. F., Pérez, H. P., Martínez, B. A., \& Vargas, V. L. (2011). Aplicación de modelos de simulación en el studio y planificación 
de la agricultura, una revisión. Tropical and Subtropical Agroecosystems, 14, 999-1010.

Conder, G. A., \& Campbell, W. C. (1995). Chemotherapy of nematode infections of veterinary importance, with special reference to drug resistance. Advances in Parasitology, 35, 1-84.

Coop, R., \& Sykes, A. (2002). Interactions between gastrointestinal parasites and nutrients. In M. Freer \& H. Dove (eds). Sheep Nutrition. Wallingford, CAB International.

Cruz, R. M., Martínez, M. I., López-Collado, J., VargasMendoza, M., González-Hernández, H., \& Fajersson, P. (2012). Effect of ivermectin on the survival and fecundity of Euoniticellus intermedius (Coleoptera: Scarabaeidae). Revista de Biología Tropical, 60, 333-345.

Encalada, M. L. A., Cordoba, B. J. A., Vargas, M. J. J., García, R. M. J., Uicab, B. L., \& Del Río, R. J. (2009). Prevalencia de nematodos gastroentéricos de becerros en sistemas de doble propósito del municipio de Escárcega, Campeche, México. Agrociencia, 43, 569-576.

Escurra, M. E. (2001). Situación de la ganadería lechera en Cajamarca. Revista de Investigaciones Veterinarias del Perú, 12, 21-26.

Fine, A., Hartman, R., Krecek, R. C., \& Groeneveld, H. (1993). Effects of time, from collection to processing, on the recovery of Haemonchus contortus third-stage larvae from herbage. Veterinary Parasitology, 51, 77-83.

Fernández, R. M., Vázquez, P. V., \& Liebano, H. E. (1994). Development and recovery of Haemonchus contortus first larval stages on experimental plots in Mexico. Veterinary Parasitology, 51, 263-269.

Flota- Bañuelos, C., López-Collado, J., Vargas-Mendoza, M., Fajersson, P., González-Hernández, H., \& Martínez-Morales, I. (2012). Efecto de la ivermectina en la dinámica espacio-temporal de escarabajos estercolero en Veracruz, México. Tropical and Subtropical Agroecosystems, 15, 227-239.

Francisco, I., Sánchez, J. A., Cortiñas, F. J., Francisco, J. R., Suárez, J., Capazal, C., Suárez, J. L., Arias, M. S., Morrondo, P., Sánchez-Andrade, R., \& Paz-Silva, A. (2011). Efficacy of ivermectin pour-on against nematodes infecting foals on pasture: coprological and biochemical analysis. Journal of Equine Veterinary Science, 31, 530-535.

García, E. (1987). Modificaciones al sistema de clasificación climática de Köppen. México, D.F.: Offset Larios. S.A.

Gruner, L. \& Sauve, C. (1982). The distribution of Trichostrongyle infective larvae on pasture and grazing behavior in calves. Veterinary Parasitology, 11, 203-213.
Giudici, C., Aumont, G., Mahieu, M., Saulai, M., \& Cabaret, J. (1999). Changes in gastrointestinal helminth species diversity in lams under mixed grazing on irrigated pastures in the tropics (French West Indies). Veterinary Research, 30, 573-581.

Iqbal, Z., Lateef, M., Khan, M. N., Muhammad, G., \& Jabbar, A. (2005). Temporal density of trichostrongylid larvae on a communal pasture in a sub-tropical region of Pakistan. Pakistan Veterinary Journal, 25, 87-91.

Jackson, F. (1993). Anthelmintic resistance- the state of play. British Veterinary Journal, 149, 123-138.

Jimenez, A. E., Montenegro, V. M., Hernández, J., Dolz, G., Maranda, L., Galindo, J., Epe, C., \& Schmieder, T. (2007). Dynamic of infections with gastrointestinal parasites and Dictyocaulus viviparus in dairy and beef cattle from Costa Rica. Veterinary Parasitology, 148, 262-271.

Krecek, R. C., Groeneveld, H. T., \& Van Wyk, J. A. (1991). Effects of time, day, season and stratum on Haemonchus contortus and Haemonchus placei third stage larvae on irrigated pasture. Veterinary Parasitology, 40, 87-98.

Krecek, R. C., \& Maingi, N. (2004). Comparison of two techniques used for the recovery of third-stage strongylid nematode larvae from herbage. Veterinary Parasitology, 122, 233-243.

Levine, N. D. (1963). Weather, climate and the bionomics of ruminant nematode larvae. Advances in Veterinary Science, 8, 215-261.

Liebano, H. E., Vázquez, P. V., \& Fernández, R. M. (1998). Sobrevivencia de larvas infectantes de Haemonchus contortus en un clima cálido subhúmedo en México. Veterinaria México, 29, 245-250.

Litell, R. C., Henry, P. R., \& Ammerman, C. B. (1998). Statistical analysis of repeated measures data using SAS procedures. Journal of Animal Science, 76, 1216-1231.

López-Collado, J. (2004). SUPRA®. Surface response program for the analysis of spatial data. Colegio de Postgraduados. Veracruz, México.

Morales, G. A., Pino, L. A., Graterol, D., \& Perdomo, L. (1991). Técnica de muestreo para la estimación de la abundancia y la prevalencia de nematodos parásitos de bovinos. Revista de la Facultad de Ciencias Veterinarias, 1, 46-50.

Morales, G. A., \& Pino, L. A. (2009). Nematodos parásitos de los rumiantes domésticos en Venezuela diagnóstico y control. Barinas, Venezuela. Ed. Laboratorio de diagnóstico veterinario ALIANI.

Nari, A., Cardozo, H., Berdié, J., Canábez, F., \& Bawen, R. (1977). Dinámica de población para nematodes gastrointestinales de ovinos en el Uruguay. Veterinaria, 14, 11-24. 
Neyman, J. (1939). On a new class of contagious distributions are applicable in entomology and bacteriology. The Annals of Mathematical Statistics, 10, 35-57.

Niec, R. (1968). Cultivo e identificación de larvas infectantes de nematodos gastroentéricos del bovino y ovino. Manual técnico 3. Instituto Nacional de Tecnología Agropecuaria. Buenos Aires, Argentina.

Niec, R. (1972). Método de recuperación de larvas infectantes de nematodos parásitos de los pastos. Revista de Medicina Veterinaria, 53, 289-293.

Olivares, P. J., Gutiérrez, S. I., \& Valencia, A. M. T. (2006). Prevalencia de nematodos gastroentéricos en terneros predestete del trópico de Guerrero, México, durante la época lluviosa. REDVET® Revista Electrónica de Veterinaria, 2, 1-5. Recuperado de http://redalyc. uaemex.mx/redalyc/pdf/636/63612653009.pdf

Oliveira, A. L. F., Costa, C., Rodella, R. A., Silva, B. F., \& Amarante, A. F. T. (2009). Effect of plant trichomes on the vertical migration of Haemonchus contortus infective larvae on five tropical forages. Tropical Animal Health and Production, 41, 775-782.

Pielou, E. C. (1960). A single mechanism to account for regular, random and aggregated populations. The Journal of Ecology, 48, 575-584.

Rege, J. E. O., Tembely, S., Mukasa-Mugewa, E., Sovani, S., Anindo, D., Lahlou-Kassi, A., Nagd, S., \& Bake, R. L. (2002). Effect of breed and season on production and response to infections with gastrointestinal nematode parasites in sheep in the highlands of Ethiopia. Livestock Production Science, 78, 159-174.

Rodriguez, R. I., Domínguez, J. L., \& Cob, L. A. (1994). Técnicas diagnósticas de parasitología veterinaria. Universidad Autónoma de Yucatán. Mérida, México.

Rojas, B. A. (1964). La binomial negativa y la estimación de intensidad de plagas en el suelo. Fitotecnia Latinoamericana, 1, 27-36.

Rossanigo, C. E., \& Gruner, L. (1994). Relative effect of temperature and moisture on the development of strongyle eggs to infective larvae in bovine pats in Argentina. Veterinary Parasitology, 55, 317-325.

Sandoval, E., Morales, G., Jiménez, D., Pino, A., \& Márquez, A. (2002). Dinámica del recuento de huevos por gramo de heces de estróngilos digestivos a diferentes horas del día en becerros naturalmente infectados. Veterinaria Tropical, 27, 51-62.

Sani, R. A., Chong, D. T., Halim, R. A., Chandrawathni, P., \& Rajamanickam, C. (1995). Control of gastrointestinal strongylosis by grazing management. Internal Conference "Novel Approaches to the Control of Helminth Parasites of Livestock". University of New England. Armidale. NSW. Australia: 61.
Saunders, L. M., Tompkins, D. M., \& Hudson, P. H. (2000). Spatial aggregation and temporal migration of freeliving stages on the parasitic nematode Trichostrongylus tenius. Functional Ecology, 14, 468-473.

Silva, B. F., Amarante, M. R. V., Kadri, S. M., CarrijoMauad, J. R., \& Amarante, A. F. T. (2010). Vertical migration of Haemonchus contortus third stage larvae on Brachiaria decumbens grass. Veterinary Parasitology, 158, 85-92.

Soulsby, E. J. L. (1987). Parasitología y enfermedades parasitarias de los animales domésticos. México, D.F.: Ediciones Nueva Interamericana.

SAS Institute (Statistical Analysis System). (2004). SAS/ STAT ${ }^{\circledR} 9.1$ User's Guide. North Carolina: SAS Institute Inc. Cary.

Steel, R. G. D., Torrie, J. H., \& Dickey, D. A. (1997). Principles and procedures of statistics a biometrical approach. New York: McGraw-Hill.

Taylor, E. L. (1939). Technique for the estimation of pasture infestation by strongyloid larvae. Parasitology, 31, 473-478.

Taylor, L. R. (1961). Aggregation, variance and the mean. Nature, 189, 732-735.

Thienpont, D., Rochette, F., \& Vanparis, O. F. J. (1986). Diagnóstico de las helmintiasis por medio del exámen coprológico. Beerse, Bélgica: Jannsen Research Foundation.

Tyrrell, K. L., Dobson, R. J., Stein, P. A., \& Walkden, S. W. (2002). The effects of ivermectin and moxidectin on egg viability and larval development of ivermectinresistant Haemonchus contortus. Veterinary Parasitology, 107, 85-93.

Uhlinger, C., Fetrow, J., \& Johnstone, C. (1988). A field evaluation of benzimidazole drugs in a herd of dairy goats. Journal of Veterinary Internal Medicine, 2, 113-116.

Vázquez-Luna, A., Pérez-Flores, L., \& Díaz-Sobac, R. (2007). Biomoléculas con actividad insecticida: una alternativa para mejorar la seguridad alimentaria. Ciencia y Tecnología Alimentaria, 5, 306-313.

Vázquez, P. V. M., Flores, C. J., Santiago, V. C., Herrera, R. D., Palacios, F. A., Liébano, H. E., \& Pelcastre, O. A. (2004). Frecuencia de nematodos gastroentéricos en bovinos de tres áreas de clima subtropical húmedo de México. Técnica Pecuaria en México, 42, 237-245.

Vera, G. J., Pinto, M. V., López, C. L., \& Reyna, R. R. (2002). Ecología de poblaciones de insectos. México, D.F.: Colegio de Postgraduados.

Waller, P. J. (2003). Global perspectives on nematode parasite control in ruminant livestock: the need to adopt alternatives to chemotherapy, with emphasis on biological control. 